Journal of Applied AnALysis

Vol. 14, No. 1 (2008), pp. 1-12

\title{
FINITE PARTITIONS OF TOPOLOGICAL GROUPS INTO CONGRUENT THICK SUBSETS
}

\author{
H. WEBER and E. ZOLI
}

Received February 28, 2006 and, in revised form, February 13, 2007

\begin{abstract}
We establish fairly general sufficient conditions for a locally compact group (a Baire topological group) to admit partitions into finitely many congruent $\mu$-thick (everywhere of second category) subsets.
\end{abstract}

\section{INTRODUCTION}

It was shown by Sierpiński [13] that a $\mathbb{Q}$-linear subspace $H$ of $\mathbb{R}$ of codimension one is a $\lambda$-thick set, in the sense that $\mathbb{R} \backslash H$ has Lebesgue inner measure zero. This of course implies that $\mathbb{R}$ can be partitioned into $\aleph_{0}$-many congruent (namely, each a translate of the other) $\lambda$-thick subsets. In [4], Erdős and Marcus generalized Sierpiński's example considerably, proving that for every cardinal number $\mathfrak{m}$ such that $\aleph_{0} \leq \mathfrak{m} \leq 2^{\aleph_{0}}$ there exists a subgroup of $\mathbb{R}$ of index $\mathfrak{m}$ that is a $\lambda$-thick set (hence there is a decomposition of $\mathbb{R}$ into $\mathfrak{m}$-many disjoint congruent $\lambda$-thick sets).

2000 Mathematics Subject Classification. Primary: 28C10, 54E52; Secondary: 20K27, 22B99.

Key words and phrases. Locally compact groups, Haar measure, Baire category, Steinhaus property, finite partitions.

ISSN 1425-6908（C) Heldermann Verlag. 
We have recently extended Erdős and Marcus' result to a rather general class of locally compact Abelian groups [14] equipped with a Haar measure $\mu$.

After dealing with the problem of splitting a locally compact group into infinitely many congruent $\mu$-thick subsets, it is natural to inquire under which conditions it admits finite partitions of the same type.

In [6, Section 16], Halmos showed that $\mathbb{R}$ is a union of two disjoint congruent $\lambda$-thick sets. More recently, Bernardi and Bondioli [2] extended Halmos' argument to locally compact groups and found sufficient conditions for them to be decomposed into $k$ disjoint congruent $\mu$-thick sets (cf. the comment at the end of Section 4).

In this paper we deal with the same type of questions as Bernardi and Bondioli [2]. In Corollary 3.6 we give a precise characterization of those locally compact Abelian groups that admit a partition into $k$ congruent $\mu$-thick subsets. We also study finite decompositions of a Baire topological group into congruent sets that are "thick" in a topological sense, i.e., everywhere of second category. To treat the two notions of $\mu$-thick sets and everywhere of second category sets simultaneously, in the spirit of [14] we first examine "thickness" with respect to a generic ideal $\mathcal{I}$ (see Definition 2.1). Both special cases we are mainly interested in are then obtained by taking for $\mathcal{I}$ the ideal of locally $\mu$-null sets or the ideal of first category sets.

We also present partial results in the noncommutative case (see Corollaries 3.7 and 3.8), which allow us to treat some classical noncommutative groups such as the group $\mathrm{GL}_{n}(\mathbb{R})$ of all regular $n \times n$ matrices over $\mathbb{R}$, and the group $\mathrm{S}_{\aleph_{0}}$ of permutations of a countably infinite set.

Throughout the paper, $G$ stands for a multiplicatively written Hausdorff topological group with neutral element $e$. If $H$ is a subgroup of $G$, we write $H \leq G$. If $A \subseteq G$, then $\langle A\rangle$ denotes the subgroup of $G$ generated by $A$. The topological closure of $A$ is denoted by $\operatorname{cl}(A)$. Two subsets $A, B$ of $G$ are said to be congruent if $A=B x$ for some $x \in G$.

\section{Thick Sets And the Steinhaus Property}

Let $X$ be a topological space, $\mathcal{B}$ its Borel $\sigma$-algebra and $\mu: \mathcal{B} \rightarrow[0, \infty]$ a Borel measure on $X$. A subset $A$ of $X$ is called $\mu$-thick if the only Borel sets contained in $X \backslash A$ are locally $\mu$-null sets. ${ }^{1}$ As observed in [14], if $X$ is decomposed into $k$ disjoint $\mu$-thick subsets $A_{1}, \ldots, A_{k}$ (with $k>1$ ), then the sets $A_{i}$ are "completely nonmeasurable" in the sense that, for every Borel set $B$ that is not a locally $\mu$-null set, $A_{i} \cap B$ is not measurable (in

\footnotetext{
${ }^{1}$ A subset $A$ of $X$ is a locally $\mu$-null set if, for every $B \in \mathcal{B}$ with $\mu(B)<\infty, A \cap B$ is contained in some $N \in \mathcal{B}$ with $\mu(N)=0$.
} 
particular, it does not belong to the $\sigma$-algebra generated by $\mathcal{B}$ and the $\sigma$ ideal $\mathcal{N}$ of all locally $\mu$-null sets).

We now give a topological concept which can be considered as the topological analogue of $\mu$-thickness. A subset $A$ of $X$ is said to be everywhere of second category (in $X$ ) if $A \cap O$ is of second category for every nonempty open subset $O$ of $X$. It turns out that $A$ is everywhere of second category iff $X \backslash A$ contains no Borel set of second category. If $X$ has a partition into $k$ everywhere of second category subsets $A_{1}, \ldots, A_{k}$ (with $k>1$ ), then for every Borel set $B$ of second category the sets $A_{i} \cap B$ do not belong to the $\sigma$-algebra generated by $\mathcal{B}$ and the $\sigma$-ideal $\mathcal{F}$ of first category subsets of $X$ (equivalently, they do not have the Baire property).

The very strict analogy between the two concepts of " $\mu$-thick sets" and "everywhere of second category sets" suggests a more general notion including both:

Definition 2.1. Let $\mathcal{I}$ be an ideal $^{2}$ on $X$. A subset $A$ of $X$ is $\mathcal{I}$-thick if the only Borel sets contained in $X \backslash A$ belong to $\mathcal{I}$.

So, "N $\mathcal{N}$-thick" means " $\mu$-thick", and " $\mathcal{F}$-thick" means precisely "everywhere of second category".

If $X$ is a topological group, then $\mathcal{F}$ is both right- and left-invariant (i.e., $F x \in \mathcal{F}$ and $x F \in \mathcal{F}$ for every $F \in \mathcal{F}$ and every $x \in X)$.

If $X$ is a locally compact group and $\mu$ a left Haar measure on $X$, i.e., a nonzero left-invariant Borel measure $\mu: \mathcal{B} \rightarrow[0, \infty]$ that is finite on compact sets, (outer) open regular on all Borel sets, and (inner) compact regular on all open sets, then also $\mathcal{N}$ is both right- and left-invariant.

The following definition is essential for our inquiry. In fact, the Steinhaus property captures the necessary interrelation between a given ideal $\mathcal{I}$ and the structure of $G$ as a topological group:

Definition 2.2. We say that an ideal $\mathcal{I}$ on $G$ has the Steinhaus property if, for every $A \in \mathcal{P}(\mathcal{G}) \backslash \mathcal{I}$ and every $B \in \mathcal{B} \backslash \mathcal{I}$, the set $A B^{-1}$ has a nonempty interior. $^{3}$

The Steinhaus property holds true in the most important cases we are interested in:

Theorem 2.3 ([1], Theorem 1). Let $G$ be locally compact and let $\mu$ be a Haar measure on $G$. Then the $\sigma$-ideal $\mathcal{N}$ of locally $\mu$-null subsets of $G$ has the Steinhaus property.

\footnotetext{
${ }^{2}$ i.e., a nonempty system of subsets of $X$ such that $A, B \in \mathcal{I}$ and $C \subseteq A \cup B$ imply $C \in \mathcal{I}$

${ }^{3}$ Note that our definition of "Steinhaus property" slightly differs from that in [3, p. 175].
} 
Theorem 2.4 ([11], Corollary 4). Let $G$ be a Baire topological group. Then the $\sigma$-ideal $\mathcal{F}$ of first category subsets of $G$ has the Steinhaus property.

The following characterization of the Steinhaus property in terms of $\mathcal{I}$ thick sets is important here:

Proposition 2.5 ([14], Proposition 3.6). An ideal $\mathcal{I}$ on $G$ has the Steinhaus property if and only if, for every $A \in \mathcal{P}(G) \backslash \mathcal{I}$ and every dense subset $D$ of $G$, the set $D A$ is $\mathcal{I}$-thick.

\section{Finite partitions into CONGRUent thick Sets}

3.1. General approach. Proposition 3.1 below serves as an illustration of the general strategy we shall follow to split $G$ into $k$ congruent subsets: it is sufficient (even necessary, if $G$ is Abelian) to find $H \leq L \leq G$ with $|L: H|=k$.

Proposition 3.1. Let $k \in \mathbb{N}$. Consider the following conditions:

(i) $G$ has an element of infinite order or a subgroup of order $k$;

(ii) there exist $H \leq L \leq G$ with $|L: H|=k$;

(iii) $G$ can be partitioned into $k$ congruent subsets.

Then (i) $\Rightarrow$ (ii) $\Rightarrow$ (iii). If $G$ is Abelian, then (iii) $\Rightarrow$ (i).

Proof. (i) $\Rightarrow$ (ii) If $G$ has an element $g$ of infinite order, put $L:=\langle g\rangle$ and $H:=\left\langle g^{k}\right\rangle$; if $G$ contains a subgroup $K$ of order $k$, then let $L:=K$ and $H:=\{0\}$.

(ii) $\Rightarrow$ (iii) Let $X$ be a system of left coset representatives of $L$ in $G$ and let $\left\{a_{1}, \ldots, a_{k}\right\}$ be a system of right coset representatives of $H$ in $L$. Then $G$ is the disjoint union of the sets $X H a_{1}, \ldots, X H a_{k}$.

Suppose now that $G$ is Abelian. We prove (iii) $\Rightarrow$ (i). Assume that there exist $A \subseteq G$ and $x_{1}, \ldots, x_{k} \in G$ so that $G$ is the disjoint union of the sets $A x_{1}, \ldots, A x_{k}$. Moreover, assume that $G$ is a torsion group. Then $K:=\left\langle x_{1}, \ldots, x_{k}\right\rangle$ is a finite subgroup of $G$. From $A x_{i} \cap K=$ $(A \cap K) x_{i}$ for all $i=1, \ldots, k$, it follows that $K$ is the disjoint union of the sets $(A \cap K) x_{1}, \ldots,(A \cap K) x_{k}$. Therefore $k$ divides $|K|$ and consequently $K$ contains a subgroup of order $k$.

Referring to the proof of (ii) $\Rightarrow$ (iii), it is important for our purpose to know what guarantees the "thickness" of the sets $X H a_{1}, \ldots, X H a_{k}$ forming the partition of $G$. Below is an initial answer to this question: 
Proposition 3.2. Let $\mathcal{I}$ be a right-invariant ideal on $G$ with the Steinhaus property and $H \leq L \leq G$ with $H \notin \mathcal{I}$ and $|L: H|=k \in \mathbb{N}$. Let $\left\{a_{1}, \ldots, a_{k}\right\}$ be a system of right coset representatives of $H$ in $L$ and $X$ a system of left coset representatives of $L$ in $G$ such that $\operatorname{cl}(X H)=G$. Then the sets $X H a_{1}, \ldots, X H a_{k}$ form a partition of $G$ into congruent $\mathcal{I}$-thick subsets.

Proof. By Proposition 2.5 (applied to $D:=X H$ and $A=H$ ), the set $X H$ is $\mathcal{I}$-thick. By invariance of $\mathcal{I}$, so are the sets $X H a_{1}, \ldots, X H a_{k}$.

In view of Proposition 3.2, we are now looking for conditions which imply that $H$ does not belong to $\mathcal{I}$ and that $X H$ is dense in $G$. The following lemma answers the latter problem, while Lemma 3.4 serves the former.

Lemma 3.3. Let $H \leq L \leq G$ with $|L: H|=k \in \mathbb{N}$. If $\operatorname{cl}(H) \supseteq L$ or $|\operatorname{cl}(H): H| \geq \aleph_{0}$, then there exists a system $X$ of left coset representatives of $L$ in $G$ such that $\operatorname{cl}(X H)=G$.

Proof. If $\operatorname{cl}(H) \supseteq L$, then for every system $X$ of left coset representatives of $L$ in $G$ we have $\operatorname{cl}(X H) \supseteq X \cdot \operatorname{cl}(H) \supseteq X L=G$.

Assume now that $|\operatorname{cl}(H): H| \geq \aleph_{0}$. Let $\left\{a_{1}, \ldots, a_{k}\right\}$ be a system of left coset representatives of $H$ in $L$. We show by induction that, for every $i=1, \ldots, k$, there is $y_{i} \in \operatorname{cl}(L)$ such that $y_{i} \cdot \operatorname{cl}(H)=a_{i} \cdot \operatorname{cl}(H)$ and $y_{i} L \cap y_{j} L=\emptyset$ if $i \neq j$. Put $y_{1}:=a_{1}$. Suppose that $1 \leq i<k$ and that $y_{1}, \ldots, y_{i}$ have already been selected in a proper way. As $|\operatorname{cl}(H): H| \geq \aleph_{0}$, it follows that

$$
a_{i+1} \cdot \operatorname{cl}(H) \nsubseteq \bigcup_{j=1}^{i} \bigcup_{r=1}^{k} y_{j} a_{r} H=\bigcup_{j=1}^{i} y_{j} L .
$$

Hence, there exists $y_{i+1} \in a_{i+1} \cdot \operatorname{cl}(H) \backslash \bigcup_{j=1}^{i} y_{j} L \subseteq \operatorname{cl}(L)$.

Let $Y$ be a system of left coset representatives of $L$ in $\operatorname{cl}(L)$ including $\left\{y_{1}, \ldots, y_{k}\right\}$ and let $Z$ be a system of left coset representatives of $\operatorname{cl}(L)$ in $G$. Then $X:=Z Y$ is a system of left coset representatives of $L$ in $G$. It remains to check that $\operatorname{cl}(X H)=G$. From

$$
\operatorname{cl}(L)=\bigcup_{i=1}^{k} \operatorname{cl}\left(a_{i} H\right)=\bigcup_{i=1}^{k} \operatorname{cl}\left(y_{i} H\right) \subseteq \operatorname{cl}(Y H)
$$

it follows that

$$
G=Z \cdot \operatorname{cl}(L) \subseteq Z \cdot \operatorname{cl}(Y H) \subseteq \operatorname{cl}(Z Y H)=\operatorname{cl}(X H) .
$$


Lemma 3.4. Let $k \in \mathbb{N}$ and let $A$ be a direct sum of cyclic groups. Assume that $A$ has an element of infinite order or a subgroup of order $k$. Then $A$ contains a subgroup of index $k$. Moreover, if $|A|>\aleph_{0}$, then for every $n \in \mathbb{N}$ there are $H_{n} \leq L_{n} \leq A$ such that $L_{n} \uparrow A,\left|L_{n}: H_{n}\right|=k$ and $\left|A: L_{n}\right|=|A|{ }^{4}$

Proof. One can decompose $A$ as $A=B \oplus C$, where $B$ is a direct sum of cyclic groups and $C \cong \mathbb{Z}$ or $C$ is a finite group containing a subgroup of order $k$. In either case, $C$ has a subgroup $H^{\prime}$ of index $k$. Thus $H:=B \oplus H^{\prime}$ has index $k$ in $A$.

Suppose now that $|A|>\aleph_{0}$. Then $B$ is a direct sum of $|A|$-many cyclic groups. A routine argument shows the existence of a sequence $B_{n}$ of subgroups of $B$ such that $B_{n} \uparrow B$ and $\left|B: B_{n}\right|=|A|$ for every $n \in \mathbb{N}$. It is clear that the groups $L_{n}:=B_{n} \oplus C$ and $H_{n}:=B_{n} \oplus H^{\prime}$ satisfy all required properties.

We are now in a position to prove the key result of the paper. Its abstract formulation will find adequate explanation and motivation in the next subsection.

Theorem 3.5. Let $k \in \mathbb{N}$. Let $\mathcal{I}_{1}, \ldots, \mathcal{I}_{m}$ be right-invariant $\sigma$-ideals on $G$ with the Steinhaus property and $G_{0} \leq G$ such that $G_{0} \notin \mathcal{I}_{i}$ for any $i=1, \ldots, m$. Moreover, let $N$ be a normal subgroup of $G_{0}$ such that $\widehat{G}_{0}:=$ $G_{0} / N$ is Abelian and contains an element of infinite order or a subgroup of order $k$.

(a) If $G_{0}$ is connected, or

(b) if $\left|\widehat{G}_{0}\right|>\aleph_{0}$ and $\left|G_{0}: G_{0} \cap M\right|<\left|\widehat{G}_{0}\right|$ for every open subgroup $M$ of $G$

then $G$ has a partition into $k$ congruent subsets, each being $\mathcal{I}_{i}$-thick for $i=1, \ldots, m$.

Proof. We split the proof into three steps.

(i) If $\widehat{G}_{0}$ contains an element $\hat{g}$ of infinite order, let $\widehat{K}:=\langle\hat{g}\rangle$; otherwise, let $\widehat{K}$ be a subgroup of $\widehat{G}_{0}$ of order $k$. According to a theorem of Kulikov (see [5, Theorem 18.4]), there exists an increasing sequence $\widehat{G}_{n}$ of subgroups of $\widehat{G}_{0}$ such that $\bigcup_{n=1}^{\infty} \widehat{G}_{n}=\widehat{G}_{0}$, where each $\widehat{G}_{n}$ is a direct sum of cyclic groups containing a maximal independent system of $\widehat{G}_{0}$. We may assume that $\widehat{G}_{1}$ contains $\widehat{K}$. Note also that, if $\left|\widehat{G}_{0}\right|>\aleph_{0}$, then $\left|\widehat{G}_{0}\right|=\left|\widehat{G}_{n}\right|$ for every $n \in \mathbb{N} .^{5}$

\footnotetext{
${ }^{4}$ We write $L_{n} \uparrow A$ to mean that $L_{n}$ is an increasing sequence with union $A$.

${ }^{5}$ Let $G$ be an uncountable Abelian group. It is well known that $|G|=\operatorname{rank}(G)$, where $\operatorname{rank}(G)$ is the cardinality of any maximal independent system of $G$ (for details, see [5, Section 16]).
} 
(ii) Let $\phi: G_{0} \rightarrow \widehat{G}_{0}=G_{0} / N$ be the canonical map. As $G_{0} \notin \mathcal{I}_{i}$ and $\mathcal{I}_{i}$ are $\sigma$-ideals for $i=1, \ldots, m$, there exists $r \in \mathbb{N}$ such that $\phi^{-1}\left(\widehat{G}_{r}\right) \notin \mathcal{I}_{i}$ for $i=1, \ldots, m$. It follows from Lemma 3.4 that there exist $\widehat{H} \leq \widehat{L} \leq \widehat{G}_{r}$ such that $|\widehat{L}: \widehat{H}|=k$ and $L:=\phi^{-1}(\widehat{L}) \notin \mathcal{I}_{i}$, hence $H:=\phi^{-1}(\widehat{H}) \notin \mathcal{I}_{i}$ for $i=1, \ldots, m$ and $|L: H|=k$. If $\left|\widehat{G}_{0}\right|>\aleph_{0}$, by Lemma 3.4 we may additionally assume that $\left|\widehat{G}_{r}: \widehat{L}\right|=\left|\widehat{G}_{r}\right|$. Observing that, by (i), $\left|\widehat{G}_{r}\right|=$ $\left|\widehat{G}_{0}\right|$, we therefore have $\left|\widehat{G}_{0}\right|=\left|\widehat{G}_{0}: \widehat{H}\right|=\left|G_{0}: H\right|$.

(iii) $\operatorname{As} \operatorname{cl}(H) \notin \mathcal{I}_{1}$, it follows from the Steinhaus property that $\operatorname{cl}(H)$ is an open subgroup of $G$. Hence, if (a) holds, $\operatorname{cl}(H) \supseteq G_{0} \supseteq L$. By Lemma 3.3, we therefore have $\operatorname{cl}(X H)=G$ for some system $X$ of left coset representatives of $L$ in $G \cdot{ }^{6}$ Let us now assume (b). We show that $|\operatorname{cl}(H): H| \geq \aleph_{0}$. This easily follows from

$$
\begin{aligned}
\left|G_{0}: G_{0} \cap \operatorname{cl}(H)\right|<\left|\widehat{G}_{0}\right|=\left|G_{0}: H\right| & =\left|G_{0}: G_{0} \cap \operatorname{cl}(H)\right| \cdot\left|G_{0} \cap \operatorname{cl}(H): H\right| \\
& \leq\left|G_{0}: G_{0} \cap \operatorname{cl}(H)\right| \cdot|\operatorname{cl}(H): H| .
\end{aligned}
$$

By Lemma 3.3 again, we can select a suitable system $X$ of left coset representatives of $L$ in $G$ such that $\operatorname{cl}(X H)=G$. An appeal to Proposition 3.2 finishes the proof.

The topological condition in (b) is rather mild. If $G$ is separable or Lindelöf (in particular, if $G$ is $\sigma$-compact), then $\left|G_{0}: G_{0} \cap M\right| \leq|G: M| \leq$ $\aleph_{0}$ for every subgroup $G_{0}$ of $G$ and for every open subgroup $M$ of $G$.

We would like to comment on the role of the commutativity assumption in Theorem 3.5, also reflected in some form or other in the following corollaries. It is Kulikov's theorem (applied to the Abelian quotient group $\widehat{G}_{0}$ ) that serves as a major ingredient of the proof of Theorem 3.5. ${ }^{7}$ But there is no analogue of Kulikov's theorem in the noncommutative case. Indeed, as Shelah [12] showed, there are uncountable groups all of whose proper subgroups are countable.

3.2. Applications. The general result of the previous subsection is now applied in the setting of locally compact groups (equipped with a Haar measure $\mu$ ) and Baire topological groups.

Corollary 3.6. Let $k \in \mathbb{N}$ and let $G$ be a nondiscrete, locally compact Abelian group. The following conditions are equivalent:

\footnotetext{
${ }^{6}$ As emphasized in the proof of Lemma 3.3, this is true for any such $X$.

${ }^{7}$ Likewise, Kulikov's theorem is an important tool in other measure-theoretical instances such as, e.g., Kharazishvili's assertion [10, pp. 112-113] that every nondiscrete locally compact Abelian group contains a Haar nonmeasurable subgroup, or the result of [14] already cited in the introduction.
} 
(i) $G$ can be partitioned into $k$ congruent subsets that are $\mu$-thick and everywhere of second category;

(ii) $G$ can be partitioned into $k$ congruent subsets;

(iii) $G$ has an element of infinite order or a subgroup of order $k$.

Proof. (i) $\Rightarrow$ (ii) is obvious and (ii) $\Rightarrow$ (iii) follows from Proposition 3.1 .

(iii) $\Rightarrow$ (i) Let $U$ be a compact neighbourhood of $e$ containing an element of infinite order or a subgroup of order $k$. We shall verify the assumptions of Theorem 3.5 for $G_{0}=\langle U\rangle, N=\{e\}, \mathcal{I}_{1}=\mathcal{N}$ and $\mathcal{I}_{2}=\mathcal{F}$ (with the notation of Theorems 2.3 and 2.4). Since $G_{0}$ is open, it belongs to neither $\mathcal{N}$ nor $\mathcal{F}$. Moreover, $\left|G_{0}: N\right|=\left|G_{0}\right|>\aleph_{0}$, since $G_{0}$ is nondiscrete and locally compact. Finally, as $G_{0}$ is $\sigma$-compact, we have $\left|G_{0}: G_{0} \cap M\right| \leq \aleph_{0}$ for every open subgroup $M$ of $G$. The conclusion now follows from Theorem 3.5.

Corollary 3.7. Suppose that the connected component $G_{0}$ of e is open, and that there is a normal subgroup $N$ of $G_{0}$ such that $G_{0} / N$ is Abelian and is not a torsion group.

(a) If $G$ is a Baire topological group, then for every $k \in \mathbb{N}$ there exists a partition of $G$ into $k$ congruent subsets that are everywhere of second category.

(b) If $G$ is locally compact, then for every $k \in \mathbb{N}$ there exists a partition of $G$ into $k$ congruent subsets that are $\mu$-thick and everywhere of second category.

Proof. Since $G_{0}$ is open, it belongs to neither $\mathcal{N}$ nor $\mathcal{F}$. Now apply Theorem 3.5.

Corollary 3.8. Let $G$ be an uncountable solvable group that is Lindelöf or separable.

(a) If $G$ is a Baire topological group, then $G$ admits for some $k \in$ $\mathbb{N} \backslash\{1\}$ a partition into $k$ congruent subsets that are everywhere of second category.

(b) If $G$ is locally compact, then $G$ admits for some $k \in \mathbb{N} \backslash\{1\}$ a partition into $k$ congruent subsets that are $\mu$-thick and everywhere of second category.

Proof. Owing to the solvability of $G$, there are $G_{0} \leq G$ and a normal subgroup $N$ of $G_{0}$ such that $\left|G: G_{0}\right| \leq \aleph_{0}$ and $G_{0} / N$ is uncountable and Abelian. Since $\left|G: G_{0}\right| \leq \aleph_{0}$, the group $G_{0}$ belongs to neither $\mathcal{N}$ nor $\mathcal{F}$. Moreover, as $G$ is Lindelöf or separable, $\left|G_{0}: G_{0} \cap M\right| \leq|G: M| \leq \aleph_{0}<$ $\left|G_{0}: N\right|$ holds for every open subgroup $M$ of $G$. Appealing to Theorem 3.5 
we conclude that, for some $k \in \mathbb{N} \backslash\{1\}$, the group $G$ can be partitioned into $k$ congruent subsets that are everywhere of second category (case (a)) and additionally $\mu$-thick (case (b)).

We close this section with some examples.

Example 3.9. Let $n \in \mathbb{N} \backslash\{1\}$ and equip the additive group $\mathbb{Z}_{n}:=\mathbb{Z} / n \mathbb{Z}$ with the discrete topology. Then $\left(\mathbb{Z}_{n}\right)^{\aleph_{0}}$ becomes a nondiscrete compact Abelian group. It follows from Corollary 3.6 that $\left(\mathbb{Z}_{n}\right)^{\aleph_{0}}$ admits a partition into $k$ congruent subsets that are $\mu$-thick and everywhere of second category iff $k$ divides some power of $n$.

Example 3.10. We show that, for every $k \in \mathbb{N}$, the group $\mathrm{GL}_{\mathrm{n}}(\mathbb{R})$ endowed with the Euclidean topology can be split into $k$ congruent subsets, each being $\mu$-thick and everywhere of second category.

To this end, we verify the assumptions of Corollary 3.7. The connected component $C$ of the identity matrix is an open subgroup of $\mathrm{GL}_{\mathrm{n}}(\mathbb{R})$, since $\mathrm{GL}_{\mathrm{n}}(\mathbb{R})$ is locally connected. Let $\phi(A):=\operatorname{det} A$ for $A \in C$. Since the range of $\phi$ is a (nontrivial) connected subgroup of $\mathbb{R}^{*}$, we have $\phi(C)=\{x \in \mathbb{R}$ : $x>0\}$. Moreover, $C / \operatorname{ker}(\phi) \cong \phi(C)$. Now apply Corollary 3.7 (b) with $G_{0}=C$ and $N=\operatorname{ker}(\phi)$.

In much the same way, one sees that for any $k \in \mathbb{N}$ the group $\mathrm{GL}_{\mathrm{n}}(\mathbb{C})$ and its subgroups $T_{n}(\mathbb{R})$ and $T_{n}(\mathbb{C})$ of upper triangular matrices over $\mathbb{R}$ and $\mathbb{C}$, respectively, can be split into $k$ congruent subsets that are $\mu$-thick and everywhere of second category.

\section{Final REMARKS}

We will briefly discuss another way to apply Proposition 2.5 for decomposing $G$.

Proposition 4.1. Let $\mathcal{I}$ be a left-invariant proper ideal on $G$ with the Steinhaus property and $H \leq L \leq G$ with $|L: H|=k \in \mathbb{N}$ and $\operatorname{cl}(H)=G$. Let $\left\{a_{1}, \ldots, a_{k}\right\}$ be a system of left coset representatives of $H$ in $L$ and $X$ a system of right coset representatives of $L$ in $G$. Then the sets $a_{1} H X, \ldots, a_{k} H X$ form a partition of $G$ into $\mathcal{I}$-thick subsets.

Proof. First observe that $H X \notin \mathcal{I}$, since $\mathcal{I}$ is a left-invariant proper ideal and $G$ is the union of the sets $a_{1} H X, \ldots, a_{k} H X$. By Proposition 2.5 (applied to $D=H$ and $A=H X$ ), the set $H X$ is $\mathcal{I}$-thick; by invariance of $\mathcal{I}$, so are the sets $a_{1} H X, \ldots, a_{k} H X$. 
An analogue result can be obtained when the assumption $|L: H|=k \in \mathbb{N}$ is replaced with $|L: H|=\aleph_{0}$ and the ideal $\mathcal{I}$ is assumed to be even a $\sigma$-ideal: if $\left\{a_{n}: n \in \mathbb{N}\right\}$ is a system of left coset representatives of $H$ in $L$, then the sets $a_{n} H X, n \in \mathbb{N}$, form a partition of $G$ into $\mathcal{I}$-thick subsets. This remark and Proposition 4.1 yield, in the special case when $G$ is locally compact and $\mathcal{I}=\mathcal{N}$ is the $\sigma$-ideal of locally $\mu$-null sets, Bernardi and Bondioli's [2, Teorema 2].

It is of interest that the partition of $\mathbb{R}$ into two congruent $\lambda$-thick subsets given by Halmos [6, Section 16] can be obtained with the aid of Proposition 4.1 in a straightforward manner: apply Proposition 4.1 with $L:=\langle\xi, 1\rangle$ and $H:=\langle\xi, 2\rangle$, where $\xi \in \mathbb{R} \backslash \mathbb{Q}$ and $\mathcal{I}$ is the $\sigma$-ideal of Lebesgue null sets in $\mathbb{R}$. Taking for $\mathcal{I}$ the $\sigma$-ideal of first category subsets of $\mathbb{R}$, one sees that the sets of the partition are also everywhere of second category. ${ }^{8}$

Corollary 4.2. Let $k \in \mathbb{N}$. Let $\mathcal{I}$ be a left-invariant proper ideal on $G$ with the Steinhaus property and $N$ a dense normal subgroup of $G$. If $G / N$ has an element of infinite order or a subgroup of order $k$, then there are $a_{1}, \ldots, a_{k} \in G$ and $A \subseteq G$ such that the sets $a_{1} A, \ldots, a_{k} A$ form a partition of $G$ into $\mathcal{I}$-thick subsets.

Proof. Let $\widehat{G}:=G / N$ and $\phi: G \rightarrow \widehat{G}$ be the canonical map. By Proposition 3.1 there are $\widehat{H} \leq \widehat{L} \leq \widehat{G}$ with $|\widehat{L}: \widehat{H}|=k$. Now apply Proposition 4.1 with $L:=\phi^{-1}(\widehat{L})$ and $H:=\phi^{-1}(\widehat{H})$.

We now give an example of a noncommutative Baire topological group to which Corollary 4.2 can be applied.

Example 4.3. Endowed with the pointwise-convergence topology, the group $S_{\aleph_{0}}$ of all permutations of the integers $\mathbb{Z}$ is a Baire topological group. We will show that, for any $k \in \mathbb{N}, \mathrm{S}_{\aleph_{0}}$ can be split into $k$ congruent subsets that are everywhere of second category. The set $F$ of all finitary permutations of $\mathbb{Z}$ (i.e., those permutations moving only finitely many elements of $\mathbb{Z}$ ) is a dense normal subgroup of $\mathrm{S}_{\aleph_{0}}$. Define $\sigma: \mathbb{Z} \rightarrow \mathbb{Z}$ by $\sigma(z)=z+1$. Then $\sigma \in \mathrm{S}_{\aleph_{0}}$ and the image of $\sigma$ under the canonical map from $\mathrm{S}_{\aleph_{0}}$ onto $\mathrm{S}_{\aleph_{0}} / \mathrm{F}$ has infinite order. By Corollary 4.2 there are $\alpha_{1}, \ldots, \alpha_{k} \in \mathrm{S}_{\aleph_{0}}$ and an everywhere of second category set $A$ such that $\mathrm{S}_{\aleph_{0}}$ is the disjoint union of the sets $\alpha_{1} A, \ldots, \alpha_{k} A$. With $B:=A^{-1}$ and $\beta_{i}:=\alpha_{i}^{-1}$, we have that $\mathrm{S}_{\aleph_{0}}$ is also the disjoint union of the everywhere of second category sets $B \beta_{1}, \ldots, B \beta_{k}$.

\footnotetext{
${ }^{8}$ Another example in $\mathbb{R}$ is considered by Wilczyński in [15] (and studied also from the Baire category viewpoint).
} 
In Corollary 3.6 we have given a satisfactory answer to the question whether a locally compact Abelian group $G$ can be decomposed into $k$ congruent "thick" sets. One might ask whether also the easier Proposition 4.1 or Corollary 4.2 can be applied to this end. It therefore becomes interesting to know when $G$ has a proper dense subgroup. A necessary and sufficient condition was established by Kabenjuk [8] and, independently, by Khan [9, Corollary 5.1]: $G$ has no proper dense subgroups iff the torsion group of $G$ is open and $p G$ is open for every prime $p$.

For noncommutative locally compact groups, much less is known. In this direction, Itzkowitz and Shakhmatov's inquiry is useful [7]. It follows from their results (in particular, cf. [7, Theorem 5]) that every compact connected nonmetrizable group has a proper dense normal subgroup.

In our terminology, [2, Teorema 1] states that a locally compact group $G$ can be decomposed into $k$ disjoint $\mathcal{N}$-thick sets, provided there exist $L \leq G$ and a normal subgroup $H$ of $L$ with $|L: H|=k$ and $\operatorname{cl}(H) \supseteq L$. While Bernardi and Bondioli's Teorema 2 and its Baire category counterpart can be deduced (through Proposition 4.1) from the Steinhaus property as introduced in Definition 2.2, their Teorema 1 cannot. Yet, a close look at their proof shows that Bernardi and Bondioli's conclusion holds, more generally, by replacing $\mathcal{N}$ with a left-invariant proper ideal $\mathcal{I}$ on a topological group $G$, provided $\mathcal{I}$ satisfies the following additional property: for every Borel set $B$ not belonging to $\mathcal{I}$, there exists a neighborhood $U$ of $e$ such that $x B \cap B \notin \mathcal{I}$ for any $x \in U{ }^{9}$ It is well known that this is true not only for the ideal of locally $\mu$-null subsets of a locally compact group, but also for the ideal of first category subsets of a Baire topological group (see, e.g., $[3$, pp. 173-174]).

\section{References}

[1] Beck, A., Corson, H. H., Simon, A. B., The interior points of the product of two subsets of a locally compact group, Proc. Amer. Math. Soc. 9 (1958), 648-652.

[2] Bernardi, M. P., Bondioli, C., Partizioni di un gruppo localmente compatto in insiemi non misurabili (Italian), Boll. Un. Mat. Ital. A (7) 6 (1992), 823-841.

[3] Cichoń, J., Kharazishvili, A. B., Wȩglorz, B., Subsets of the Real Line I, Łódź University Press, Łódź, 1995.

[4] Erdős, P., Marcus, S., Sur la décomposition de l'espace Euclidien en ensembles homogènes (French), Acta Math. Acad. Sci. Hungar. 8 (1957), 443-452.

[5] Fuchs, L., Infinite Abelian Groups. Vol. I, Pure Appl. Math. 36-II, Academic Press, New York-London, 1970.

[6] Halmos, P., Measure Theory, Springer-Verlag, New York, 1974.

\footnotetext{
${ }^{9}$ Note that this property for $\mathcal{I}$ is stronger than the "Steinhaus property" defined in [3, p. 175].
} 
[7] Itzkowitz, G. L., Shakhmatov, D., Haar nonmeasurable partitions of compact groups, Tsukuba J. Math. 21 (1997), 251-262.

[8] Kabenjuk, M. I., Dense subgroups of locally compact abelian groups, Sibirsk. Mat. Zh. 21 (1980), 202-203.

[9] Khan, M. A., Chain conditions on subgroups of LCA groups, Pacific J. Math. 86 (1980), 517-534.

[10] Kharazishvili, A. B., Selected Topics of Point Set Theory, Łódź University Press, Łódź, 1996.

[11] McShane, E. J., Images of sets satisfying the condition of Baire, Ann. of Math. (2) 51 (1950), 380-386.

[12] Shelah, S., On a problem of Kurosh, Jonsson groups, and applications, in "Word Problems, II" (Conf. on Decision Problems in Algebra, Oxford, 1976), Stud. Logic Found. Math. 95, North-Holland, Amsterdam, 1980, 373-394.

[13] Sierpiński, W., Sur la question de la mesurabilité de la base de M. Hamel (French), Fund. Math. 1 (1920), 105-111.

[14] Weber, H., Zoli, E., On the Steinhaus property in topological groups, Topology Appl. 153 (2006), 2035-2046.

[15] Wilczyński, W., Finite partitions of the real line consisting of similar sets, Acta Sci. Math. (Szeged) 38 (1976), 191-192.

HANS WEBER

ENRICO ZOLI

DipARTIMENTO Di MATEMATICA ED INFORMATICA

Università DEGLI StUdi DI Udine

Via Ballanti GraZiani 33

VIA DELLE SCIENZE 206

FAENZA (RA) 48018, ITALY

UdINE 33100, ITALY

E-MAIL:ZOLI@MATH.UNIFI.IT

E-MAIL:WEBER@DIMI.UNIUD.IT 